\title{
A handy guide to science-communication theory and practice
}

Book

\section{Reviewed by}

Abstract

Keywords

The Handbook itself
M. Bucchi And B. Trench, eds. (2014). Routledge Handbook of Public Communication of SCIENCE AND TECHNOlogy. SECONd Edition. London, U.K.: ROUTLEDGE

\section{Achintya Rao}

The ever-changing nature of academic science communication discourse can make it challenging for those not intimately associated with the field scientists and science-communication practitioners or new-comers to the field such as graduate students - to keep up with the research. This collection of articles provides a comprehensive overview of the subject and serves as a thorough reference book for students and practitioners of science communication.

Public understanding of science and technology; Representations of science and technology; Scholarly communication

The first edition of the Routledge Handbook of Public Communication of Science and Technology (henceforth referred to as "the Handbook") was reviewed in a previous edition of JCOM [Delfanti, 2008]. The second edition proves to be as insightful, thought provoking and well structured as its predecessor, while broadening its international perspectives on the theory and practice of science communication. This review is divided into two sections: the first address the structure of the Handbook and its contents, and the second provides the reviewer's reflections.

Six years - the duration between the first and second editions of the Handbook can be an eternity in academia. The contents of the second edition have been appropriately updated to reflect the changes in the science-communication landscape that have taken place in the interim, in particular the strengthening of the "public engagement" paradigm. Readers would do well to begin their exploration of the Handbook with the introductory chapter written by the editors, which articulates this shift towards "public engagement" from previous models of science communication.

One of the first things a reader will notice when attempting to compare the Indices of the first and second editions of the Handbook is the expanded international scope 
of the content; to quote the editors, "... specific chapters on developing countries and on the Internet [from the first edition] have given way to a broader treatment of globalisation and the consideration in almost all chapters of applications and implications of online media...". Now, most discourse on science communication tends to come with a "Western" flavour containing certain socio-cultural beliefs and pre-suppositions - indeed the authors of all the chapters are themselves from (or based in) European or North American nations - so this attempt at addressing other perspectives and attitudes is crucial to having a truly global conversation around science communication. Fortunately for us, the editors are all too aware of this - "[the global nature of science communication] highlights how difficult and even misleading it would be to expect a single, straightforward response to contemporary challenges of science communication [...] or to fulfil the expectation of eventually finding the best and most appropriate, one-size-fits-all model of science/public interaction" - and perhaps future editions of this valuable and widely read book will include contributions from a more diverse set of authors.

Another welcome change, at least from a student's perspective, has been the inclusion of questions at the end of each chapter. The Handbook itself provides the reader with many opportunities to reflect on its content, but the questions help guide a student's line of reasoning and reflection.

The modular nature of the chapters, each written by different (groups of) experts, makes it easy for readers to dive right into the Handbook by exploring the topic of their choice. The chapters cover a rich variety of themes one would encounter in studying science communication: vectors of engagement (books, museums, film), policy (public relations, participation), actors (scientists, journalists, publics), "hot-button" issues (climate, health) as well as methodology (surveys, assessment). While the Handbook caters mainly to new-comers to the field, one of its main strengths lies in the depth of references included with each chapter: even if the reader is somewhat familiar with the topic being addressed, there are adequate pointers for further reading. However, readers should note that although the language encountered throughout the Handbook is clear and precise, it can be intimidating in places: a lack of contextual definitions of academic terminology may impede fluent, straightforward reading.

Given the complexity of the themes covered in the Handbook, it is by no means intended for casual or rapid reading. That said, the conversational style employed by some of the authors makes for very engaging reading. Over the course of my research, I have found myself returning to previously read chapters and sections in order to clarify my own line of thought. On more than one occasion I turned to the Handbook just to consult the references at the end of chapters pertaining to my area of study. It has proven to be a very valuable resource indeed!

One aspect that I found a little lacking was the diversity of science domains covered: although the editors state emphatically that it is "problematic to continue using traditional expressions like scientific community, implying internal homogeneity and a shared commitment to specific norms and values..." they nonetheless only afford the aforementioned "hot-button" issues their own chapters. To my mind, certain domains of science lend themselves more easily to "public engagement", perhaps due to their direct or immediate impact on broader society; 
think climate change or GMOs. Other - possibly esoteric - domains of research, less so; think theoretical particle physics or network topology. These, in some sense less-accessible, areas of research present their own science-communication challenges, and discourse that both contextualises these challenges and proposes ideas for facing them would benefit a large number of academics and practitioners.

Nevertheless, the Handbook holds open a captivating door into the world of science communication and makes for an excellent point-of-entry for those wishing to explore this field of research. Every university library would do well to have a copy in stock for its researchers as well as its students.

\section{References}

Author

\section{How to cite}

Delfanti, A. (2008). 'How-to establish PCST. Two handbooks on science communication'. JCOM 7 (4), R01. URL: http://jcom.sissa.it/archive/07/04/Jcom0704\%282008\%29R01.

Achintya Rao is a professional science communicator working for the CMS (Compact Muon Solenoid) Collaboration at the Large Hadron Collider and is based at CERN in Geneva, Switzerland. Since January 2014, he has also been a part-time Ph.D. student at the Science Communication Unit of the University of the West of England in Bristol, U.K. . His research focuses on outreach and communication practices in the field of particle physics, specifically in the context of CERN. He can be found online at http:/ / achintyarao.in. E-mail: achintya.rao@cern.ch.

Rao, A. (2015). 'A handy guide to science-communication theory and practice'. JCOM 14 (04), R01. 